\title{
Spintronics: Overview on Spin Based Electronics and Its Potential Applications
}

\author{
Bushra Irfan \\ Department of Physics, Aligarh Muslim University (AMU), Aligarh, India \\ Email address: \\ bushra.ph@amu.ac.in \\ To cite this article: \\ Bushra Irfan. Spintronics: Overview on Spin Based Electronics and Its Potential Applications. International Journal of Materials Science \\ and Applications. Vol. 0, No. 5, 2021, pp. 94-97. doi: 10.11648/j.ijmsa.20211005.11
}

Received: August 14, 2021; Accepted: September 3, 2021; Published: September 11, 2021

\begin{abstract}
Spintronics is a branch of electronics that utilizes the spin of an electron to carry information. Spin is a quantum phenomenon and attracted researchers because it is an ideal way for representing logic " 0 " and " 1 " (used in electronics) with spin pointing "up" or "down" with respect to a magnetic field. Therefore, spin imparts itself into a new kind of binary logic of "one" and "zero". These characteristics open a new possibility of spintronics application in various fields such as magnetic storage technology and quantum computers. Spintronics is an emerging field for next-generation nanoelectronic devices to minimize their power consumption (which is the major issue in future microelectronics technology) and increase memory capabilities. This article briefly introduces the fundamentals of spintronics, progress in spintronics and its applications; it also features the current trend and challenging goal in this area.
\end{abstract}

Keywords: Spintronics, Spin Relaxation, Spin Transfer Torque (STT)

\section{Introduction}

Spintronics (or spin electronics) is an expanding field, and as the name suggests, it exploits the spin properties of an electron in extension to charge degree of freedom. Our present-day electronics almost rely exclusively on a charge of electrons; the questions then arise "why we need spintronics?". In 1965 Gorden Moore explained how the number of components could be crammed into an integrated circuit double every year, also known as Moore's law [1]. However, Moore's law has limitations [2]; the transistor and other electronic components have reached nano-scale dimensions. Further reducing the size would lead to sweltering heat, making the circuit inoperable. Also, at the nano-scale dimension, the quantum effect comes into play; thus cannot reduce the size of the transistor or other components beyond the limit. Hence, the urge for further research considered spintronics with an immense potential to provide high speed, low power, and high-density logic and electronic memory devices.

Spintronics exploits both the intrinsic spin of an electron and its associated magnetic moment (that makes the electron act like a tiny magnet) in addition to its fundamental electronic charge [3-5]. Moreover, by utilizing the intrinsic magnetic moment of electrons, which arises from its angular momentum or "spin", spintronic devices are produced with smaller size and more versatile characteristics than traditional electronics devices $[6,7]$. The exclusive property of spin is its coherence or relaxation time. Spin relaxation is a property in which spin tends to stay in its state for a long time, unlike charge, which can easily change its state by scattering or collision with impurities or other charges. This property expands spintronics application from non-volatile magnetic memories (MRAM) to Tera-Hertz devices and recently accomplish as a quantum bit in a computer as quantum computers [8-11]. However, there is an important challenge in spintronics technology to preserve coherence for a relatively long time; as longer the spin lifetime, the better and reliable the spintronic device will perform $[12,13]$.

\section{Shift from Electronics to Spintronics Prospect Faster Data Transfer}

We know the electronics depends on measuring the tiny electrical charge of electrons flowing through an electronic circuit. Thus, adding a spin degree of freedom provides new effects, new potency, and new functionalities to electronics 
termed as spintronics. Spintronics works in same way as traditional electronics that uses "zero" and "one" to represent information. The two spin states are used to represent the same binary data in spintronics. Information is saved into a spin as one of two possible orientations, as shown in Figure 1 (a). All spintronic devices act according to a simple scheme as shown in Figure 1 (b): information is stored (written) into spins as a particular spin orientation (up or down), the spins, being attached to mobile electrons, carry the information along a wire, and the information is read at a terminal [6]. Spintronics is faster and effective technology due to its spin being able to change quickly and over less power [14]. Thus, Spin states can be set quickly, which helps to transfers data promptly. The non-volatile memory, such as magnetic random access memories (MRAM), works on the principle of spintronics, has replaced several semiconductor-based memory technologies due to relatively low power consumption and spontaneously ON capability. Spintronics field has further developed Spin Transfer Torque

(a)

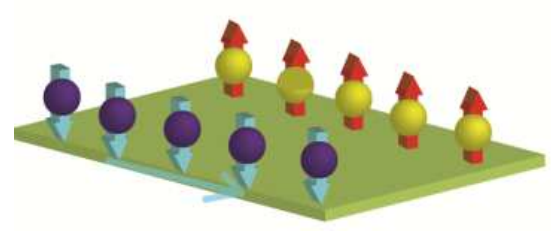

(STT) based MRAM, i.e., STT-MRAM [15, 16], which uses electric current to manipulate and switch the magnetic moment. In spin-transfer torque, torque is exerted on a ferromagnetic electrode in magnetic tunnel junction (MTJ)-consist of two ferromagnetic layers separated by non-magnetic layer [17, 18]. When an electric current flows through MTJ, the STT may cause either magnetization precession in ferromagnetic (FM) layer or reversal of magnetization [19, 20].

The spin transfer torque works by transferring spin angular momentum between two non-collinear magnetic layers (Figure 2). However, STTMRAM has the dielectric breakdown of the tunnel barrier when a large current (longitudinal) flows through the circuit and thus damage the device. To overcome this issue, one of the method is to use materials that have high spin orbit coupling such as topological insulators [21-25] with ferromagnetic layer where lateral current is used to generate spin torque [26, 27].

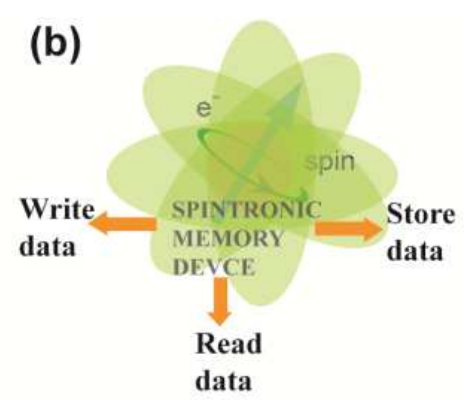

Figure 1. (a) An artistic depiction for spin degree of freedom with up and down states. (b) Spin memory device: Three important parameters of memory devices are read, write and store data.
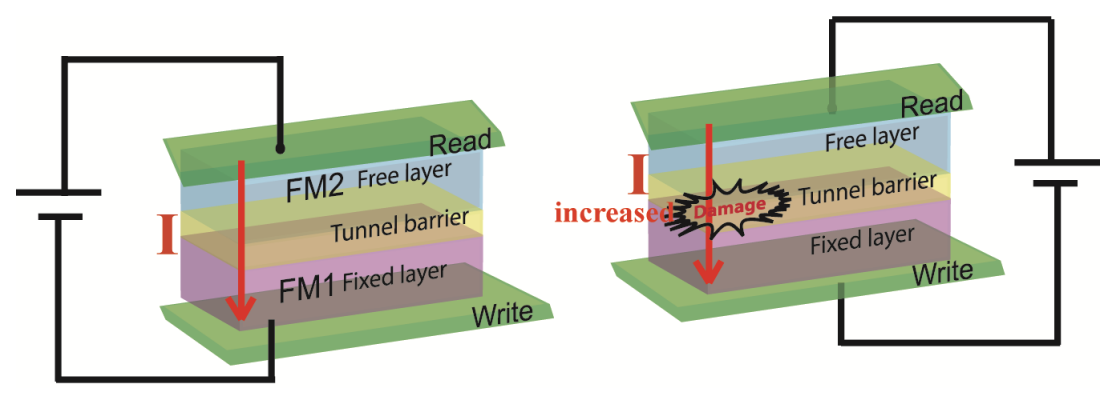

Figure 2. Schematic of spin transfer torque in multilayered structure. A spin-polarized current, coming from the bottom layer FM1 (i.e. fixed layer), induces a spin-transfer torque on top magnetic layer FM2 (free layer). If high current ows across STT then there is possibility of device to get damage.

\section{Applications of Spintronics}

Spintronics applications (Figure 3) have advanced in various fields [28-30]. Thus, instigate magnetic recording technology (1988) and benefits the spintronics progress by allowing the development of extremely sensitive nanoscale magnetic field sensors based on giant magnetoresistance (GMR) spin-valves [31, 32] and later on tunnel magnetoresistance (TMR) [33, 34]. In 2017, Samsung Electronics announced its first mass production of STTMRAM (spintransfer torque magneto resistive random access memory) that utilizes the spin-polarized current $[16,36]$. This adoption of spintronics technology by the microelectronics industry will significantly help to mature spintronics.
Nonvolatile (NV) memory (a type of computer memory that hold data even power is turned off) is a fundamental element for future high-performance microelectronics with low power consumption. Among the proposed NV memories, spintronics-based ones are particularly fascinating for applications, owing to their low voltage operation, high speed in addition to their high persistence feature [37-39]. Spintronics devices can be classified in to two categories with different writing schemes: spin-transfer torque (STT) and spin-orbit torque (SOT). The NV memories using STT have been attracted and developed most actively and are about to enter the market. On the other hand, the development of SOT is in progress [40, 41]. 


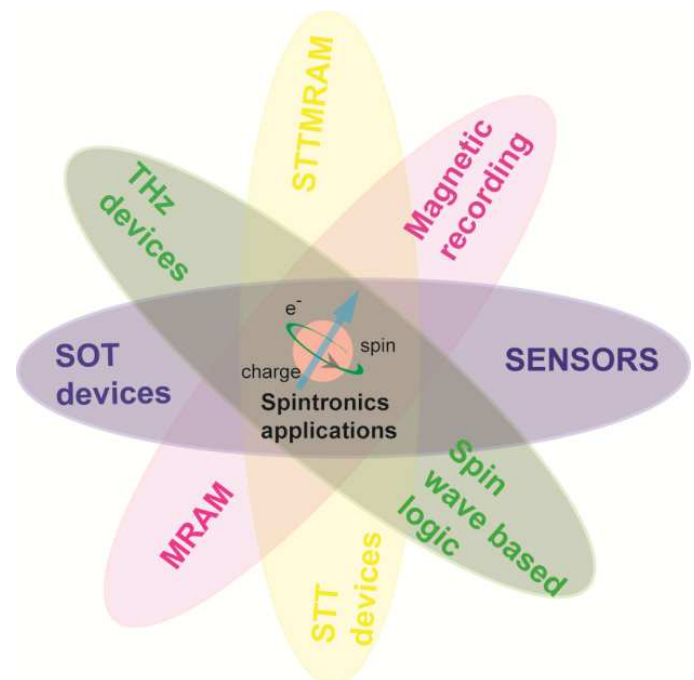

Figure 3. Present and future applications of spintronic devices.

Another application of spintronics is in TeraHertz (THz) devices and sensors $[42,43]$. Using $\mathrm{THz}$ spintronics devices, one could open new channel in computational speeds by combining ultrafast optics and photonics [41, 44]. $\mathrm{THz}$ spintronics technologies are attractive for ultrafast-computing [46]. Next are spintronic sensors with superb measuring abilities due to their small size and low cost [47, 48]. Hence, they can be used as common magnetic field sensors in electrical sensing, transmission and distribution lines monitoring vehicle detection, and bio-detection [49, 50]. Moreover, the spin of an electron can be connected to optics via photon helicity and is expected to facilitate much faster data transfer with various exciting phenomena such as spinpolarized solar battery [51] and spin-polarized solar cells. These promising new effects open up a field of exciting investigations and applications in the field of spintronics.

\section{Conclusion}

Spin-based electronics emerged as the most attractive branch in nanotechnology due to effective manipulation of spin degree of freedom in addition to their electronic charge used in traditional semiconductor electronics. The range of spintronics applications is widening from read-head devices to non-volatile magnetic memories to spin-transfer nanooscillators for future application in wireless telecommunications and future cause of an individual electron spin system as a quantum bit in a quantum computer.

\section{References}

[1] Waldrop M M; Nature, 2016530 144-147. DOI: 10.1038/530144a.

[2] Kim N S, Austin T, Blaauw D, Mudge T, Flautner K, Hu J S, Irwin M J, Kandemir M, and Narayanan V; IEEE Computer, 200336 68-75. DOI: 10.1109/MC.2003.1250885.

[3] Sarma S S; Hwang E H and Kaminski A; Solid State Commun., 2002, 127, 99-107. DOI: 10.1016/S0038-1098(03)00337-5.
[4] Lu J W, Chen E, Kabir M, Stan M R and Wolf S A; International Mater. Rev., 2016, 61, 456-472. DOI: 10.1080/09506608.2016.1204097.

[5] Salahuddin S; Nature, 2013, 494, 43-44. DOI: 10.1038/nature11944.

[6] Zutic I, Fabin J and Sharma S D; Rev. Mod. Phys. 2004, 76, 323. DOI: 10.1103/RevModPhys.76.323.

[7] Wolf S A, Awschalom D D, Buhrman R A, Daughton J M, Molnar S V, Roukes M L, Chtchelkanova A Y, Treger D M; Science, 2001, 294, 1488-1495. DOI: 10.1126/science.1065389.

[8] Vincenzo D P; Science, 1995, 270, 255-261. DOI: 10.1126/science. 270.5234 .255 .

[9] Coronado E and Epestin E J; J. Mater. Chem., 2009, 19, 16701671. DOI: $10.1039 / \mathrm{B} 901955 \mathrm{~N}$.

[10] Hirohata A, Yamada K, Nakatani Y, Prejbeanu I-L, Dieny B, Pirro P, Hille- brands B; J. Magnet. and Magnetic Mater., 2020, 509, 166711. DOI: 10.1016/j.jmmm.2020.166711.

[11] Sarma S D, Fabian J, Hu X, Zutic I; Solid State Commun., 2001, 119, 207-215. DOI: 10.1016/S0038-1098(01)00111-9.

[12] Fabian J and Sarma S D; J. Vac. Sci. and Technol. B, 1999, 17, 1708. DOI: $10.1116 / 1.590813$.

[13] Prinz A G; Phys. Today, 1995, 48, 4. DOI: 10.1063/1.881459.

[14] Ghazaly A. El, Gorchon J, Wilson R B, Pattabi A, Bokor J; J. Magn. Magn. Mater., 2020, 502, 166478. DOI: 10.1016/j.jmmm.2020.166478.

[15] Kent A D and Worledge D C; Nat. Nanotechnol., 2015, 10, 187-191. DOI: 10.1038/nnano.2015.24.

[16] Bhatti S, Sbiaa R, Hirohata A, Ohno H, Fukami S and, Piramanayagam S N; Mater. Today, 2017, 20, 530-548. DOI: https://doi.org/10.1016/j.mattod.2017.07.007

[17] Zhu J-G, Park C; Mater. Today, 2006, 9, 36-45. DOI: https://doi.org/10.1016/S1369-7021(06)71693-5

[18] Dankert A, Kamalakar M V, Wajid A, Patel R S and Dash S P; Nano Res., 2015, 8, 1357-1364 DOI: https://doi.org/10.1007/s12274-014-0627-4

[19] Ralph D C, Stiles M D; J. Magn. Magn. Mater., 2008, 320, 1190-1216. DOI: https://doi.org/10.1016/j.jmmm.2007.12.019

[20] Liu L, Lee O J, Gudmundsen T J, Ralph D C, and Buhrman R A; Phys. Rev. Lett., 2012, 109, 096602. DOI: https://doi.org/10.1103/PhysRevLett.109.096602

[21] Dankert A, Geurs J, Kamalakar M V, Charpentier S and Dash S P; Nano Lett., 2015, 15, 7976-7981. DOI: https://doi.org/10.1021/acs.nanolett.5b03080

[22] Irfan B and Chatterjee R; Appl. Phys. Lett., 2015, 107, 173108. DOI: https://doi.org/10.1063/1.4934569

[23] Irfan B; Appl. Phys. A, 2020, 126, 1-7. DOI: https://doi.org/10.1007/s00339-020-3345-7

[24] Irfan B, Joshi B P, Thamizhavel A, Deshmukh M M, Chatterjee R; Solid State Commun., 2015, 220, 45-48. DOI: https://doi.org/10.1016/j.ssc.2015.07.007

[25] Fan Y and Wang K L; World Scientif. Publishing, 2016, 6, 1640001. DOI: https://doi.org/10.1142/S2010324716400014 
[26] Chernyshov A, Overby M, Liu X, Furdyna J K, Geller Y L and Rokhinson L P; Nat. Phys., 2009, 5, 656. DOI: https://doi.org/10.1038/nphys 1362

[27] Garello K, Miron I M, Avci C O, Freimuth F, Mokrousov Y, Blugel S, Boulle O, Gaudin G, Gambardella P; Nat. Nanotech., 2013, 8, 587. DOI: https://doi.org/10.1038/nnano.2013.145

[28] Mishra R and Yang H; IEEE Trans. on Magn., 2021, 57, 1-34. DOI: https://doi.org/10.1109/TMAG.2020.3032099

[29] Mishra R, Kumar D and Yang H; Phys. Rev. Appl., 2019, 11, 054065. DOI: https://doi.org/10.1103/PhysRevApplied.11.054065

[30] Guo L, Gu X, Zhu X, Sun X; Adv. Mater., 2019, 31, 1805355. DOI: https://doi.org/10.1002/adma.201805355

[31] Yakout S M; J. Supercond. Nov Magn., 2020, 33, 2557-2580. DOI: https://doi.org/10.1007/s10948-020-05545-8

[32] Chappert C, Fert A. and Dau F N V; Nature Mater., 2007, 6, 813-823. DOI: https://doi.org/10.1038/nmat2024

[33] Hirohata A and Takanashi K; J. Phys. D: Appl. Phys., 2014, 47, 193001. DOI: 10.1088/0022-3727/47/19/193001.

[34] Peng S Z, Zhang Y, Wang M X, Zhang Y G and Zhao W; Wiley Encyclopedia of Electrical and Electronics Engineering (John Wiley and Sons, Inc., 2014) 2014, 24, 17616. DOI: https://doi.org/10.1002/047134608X.W8231

[35] Eschrig M; Rep. Prog. Phys., 2015, 78, 104501. DOI: 10.1088/0034-4885/78/10/104501.

[36] Wolf S A and Treger D; IEEE Trans. Magn., 2000, 36, 2748. DOI: https://doi.org/10.1109/20.908580

[37] Prinz G A; Science, 1998, 282, 1660. DOI: 10.1126/science. 282.5394 .1660 .

[38] Joshi V K; Engineering Science and Technology, an International Journal, 2016, 19, 1503-1513. DOI: https://doi.org/10.1016/j.jestch.2016.05.002
[39] Ramaswamy R, Lee J M, Cai K and Yang H; Appl. Phys. Rev., 2018, 5, 031107. DOI: https://doi.org/10.1063/1.5041793

[40] Endoh T and Honjo H; J. Low Power Electron. Appl., 2018, 8, 44. DOI: https://doi.org/10.3390/jlpea8040044

[41] Walowski J and Munzenberg M; J. Appl. Phys., 2016, 120, 140901. DOI: https://doi.org/10.1063/1.4958846

[42] Bocklage L; Phys. Rev. Lett., 2017, 118, 257202. DOI: https://doi.org/10.1103/PhysRevLett.118.257202

[43] Bulgarevich D S, Akamine Y, Talara M, Mag-usara V, Kitahara H, Kato H, Shiihara M, Tani M and Watanabe M; Sci. Rep,. 2020, 10, 1158. DOI: https://doi.org/10.1038/s41598-020-58085-5

[44] Feng Z, Yu R, Zhou Y, Lu H, Tan W, Deng H, Liu Q, Zhai Z, Zhu L, Cai J, Miao B and Ding H; Adv. Opt. Mater., 2018, 6, 1800965. DOI: https://doi.org/10.1002/adom.201800965

[45] Bandyopadhyay S and Cahay M; Physica E, 2005, 27, 98-103. DOI: https://doi.org/10.1016/j.physe.2004.10.012

[46] Freitas P P, Ferreira R and Cardoso S; Proc. IEEE, 2016, 104, 1894-1918. DOI: https://doi.org/10.1109/JPROC.2016.2578303

[47] Liu X, Lam K H, Zhu K, Zheng C, Li X, Du Y, Liu C and Pong P W T; IEEE Trans. On Magn., 2019, 55, 1-22. DOI: https://doi.org/10.1109/TMAG.2019.2927457

[48] Wang Y, Li J, Viehland D; Mater. Today, 2014, 17, 269-275. DOI: https://doi.org/10.1016/j.mattod.2014.05.004

[49] Zutic I, Fabian J and Sarma S D; Appl. Phys. Lett., 2001, 79, 1558. DOI: https://doi.org/10.1063/1.1399002

[50] Zutic I, Fabian J and Sarma S D; Phys. Rev. B, 2001, 64, 121201 (R).

DOI: https://doi.org/10.1103/PhysRevB.64.121201

[51] Endres B, Ciorga M, Schmid M, Utz M, Bougeard D, Weiss D, Bayreuther G and Back C H; Nat. Commun., 2013, 4, 2068. DOI: https://doi.org/10.1038/ncomms3068 